\title{
In-Lens Cryo-HRSEM of a Freeze-Dried Coiled-coil Protein Assembly
}

\author{
S.N. Dublin, V.P. Conticello, and R.P Apkarian
}

Department of Chemistry \& the Integrated Microscopy \& Microanalytical Facility, Emory University, Atlanta, GA 30322

The coiled-coil motif, composed of two or more supercoiled $\alpha$-helical peptides, is a valuable template in the de novo design of novel peptide assemblies. Extensive studies of heptad repeat sequences ( $a b c d e f g$ ) in coiled-coil systems have demonstrated that core $a / d$ positions, together with solvent-exposed $e / g$ positions, determine self-assembly properties such as strand oligomerization, orientation and thermodynamic stability.

The TZH1 peptide was designed as a 41-residue peptide sequence containing 6 heptad repeats. A mechanism for controlling peptide self-assembly was introduced into the system through the incorporation of a histidine residue at core $d$ positions in alternating heptads [1]. Acidic assembly conditions result in the protonation of proximal imidazole side-chains, introducing a destructive, electrostatic repulsion within the hydrophobic core. Attempts at visualizing TZH1 fibrils assembled in $\mathrm{NaH}_{2} \mathrm{PO}_{4}(\mathrm{pH} 4.0)$ via transmission electron microscopy (TEM) confirmed the absence of $\alpha$-helical self-assembly observed by circular dichroism (CD). TZH1 assembled under basic conditions ( $\mathrm{pH} 8.0$ ) in the same buffer exhibited helical peptide formation, as demonstrated by CD and TEM. However, the average diameter of TZH1 fibrils was greater than an order of magnitude larger than predicted.

Low temperature (LT) cryo high resolution scanning electron microscopy (cryo-HRSEM) was utilized in hopes of revealing the $\sim 3 \mathrm{~nm}$ coiled-coil protein assemblies. This technique uses high signal-to-noise ratio and SE-I image contrast to provide nanometer resolution of macromolecular structure, as demonstrated in previous structural studies of fibronectin fibrils and elastin-mimetic hydrogels $[2,3,4]$.

A $1 \mathrm{mg} / \mathrm{mL}$ protein solution $(10 \mu \mathrm{L})$ of TZH1 assembled in $10 \mathrm{mM} \mathrm{NaH}_{2} \mathrm{PO}_{4}$ buffer was applied to a 200 mesh, carbon-coated copper grid. Excess solution was wicked away with filter paper, leaving a thin aqueous film $(\sim 100 \mathrm{~nm})$. Cryo-immobilization was achieved through plunge-cooling the sample in liquid ethane $\left(-183.3^{\circ} \mathrm{C}\right)$. The grid was rapidly loaded into a Gatan CT 3500 cryostage pre-cooled to $-170{ }^{\circ} \mathrm{C}$ and transferred to the Denton DV-602 chromium coater. After achieving a vacuum of $4 \times 10^{-7}$ torr, the sample temperature was raised to $-85^{\circ} \mathrm{C}$ and held for 5 minutes to sublime bulk LT water past the hydration shell. Finally, a thin $(2 \mathrm{~nm})$ continuous chromium film was sputter coated on the sample and the stage was transferred from the coater to the the upper stage of a DS-130 FESEM for imaging. All TEM images were obtained on a JEOL LaB 6 JEM-1210 at $90 \mathrm{kV}$ accelerating voltage.

Cryo HRSEM micrographs of freeze-dried thin specimens revealed two distinct fibril morphologies. Intermediate magnification showed smooth fibril ropes $120-130 \mathrm{~nm}$ in diameter, entangled in a proteinatious mesh of fine filaments (Fig 1). Diameter measurements of the mesh components at high magnification ranged from 8-12 nm, which was within the acceptable range of a trimeric coiled-coil assembly. Included in the measurements of the protein fibrils were residual hydration shell left by conservative freeze-drying times and a $2 \mathrm{~nm}$ layer of $\mathrm{Cr}$. Confirmation of the $<4 \mathrm{~nm}$ 
filaments by LT cryoHRSEM inspired adjustments in TEM sample preparation, leading to their successful negative stain imaging using a 1:1 NanoVan /NanoW (Nanoprobes, Yaphank, NY) negative stain mixture (Fig 2).

References

[1] V.P. Conticello, et al., JACS (in press)

[2] Y. Chen et al., J of Microscopy 179 Pt.1 (1995) 67.

[3] V.P. Conticello. et al., Macromolecules 3226 (1999) 9067.

[4] R.P. Apkarian, et al., Microsc. and Microanal. 9 (2003) 286.
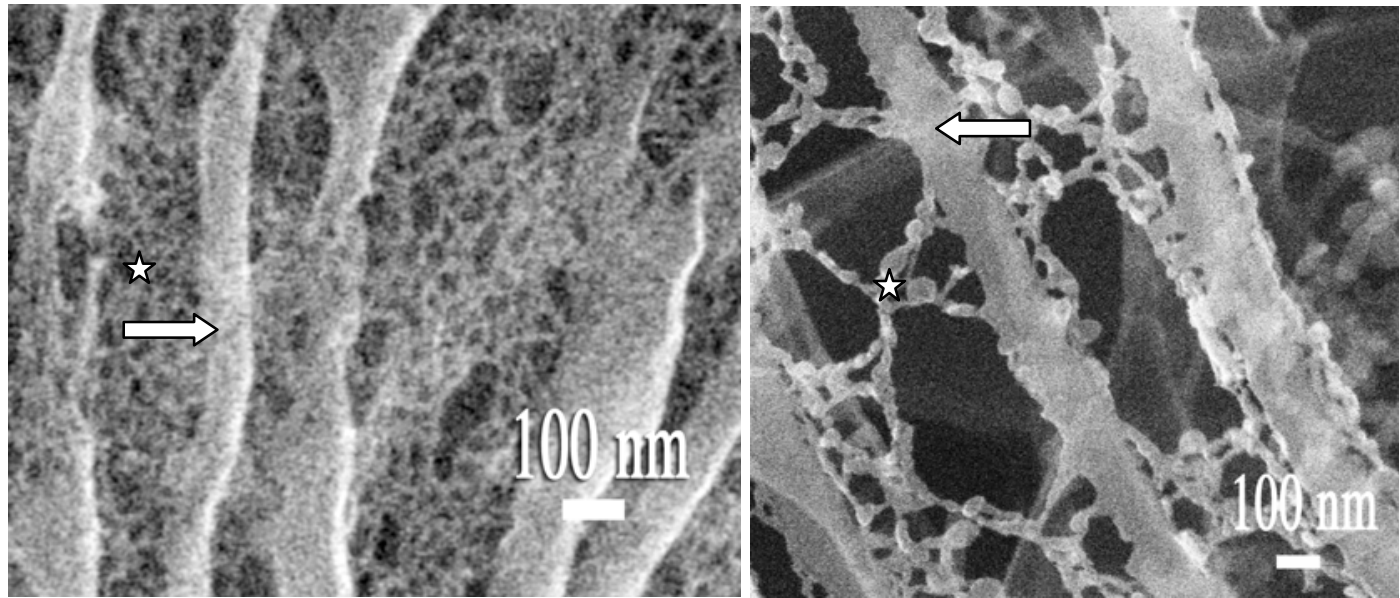

Fig.1. LT cryo-HRSEM reveals proteinatous network (stars) composed of $\sim 10 \mathrm{~nm}$ filaments interlaced with large $>100 \mathrm{~nm}$ fibril ropes (arrows).
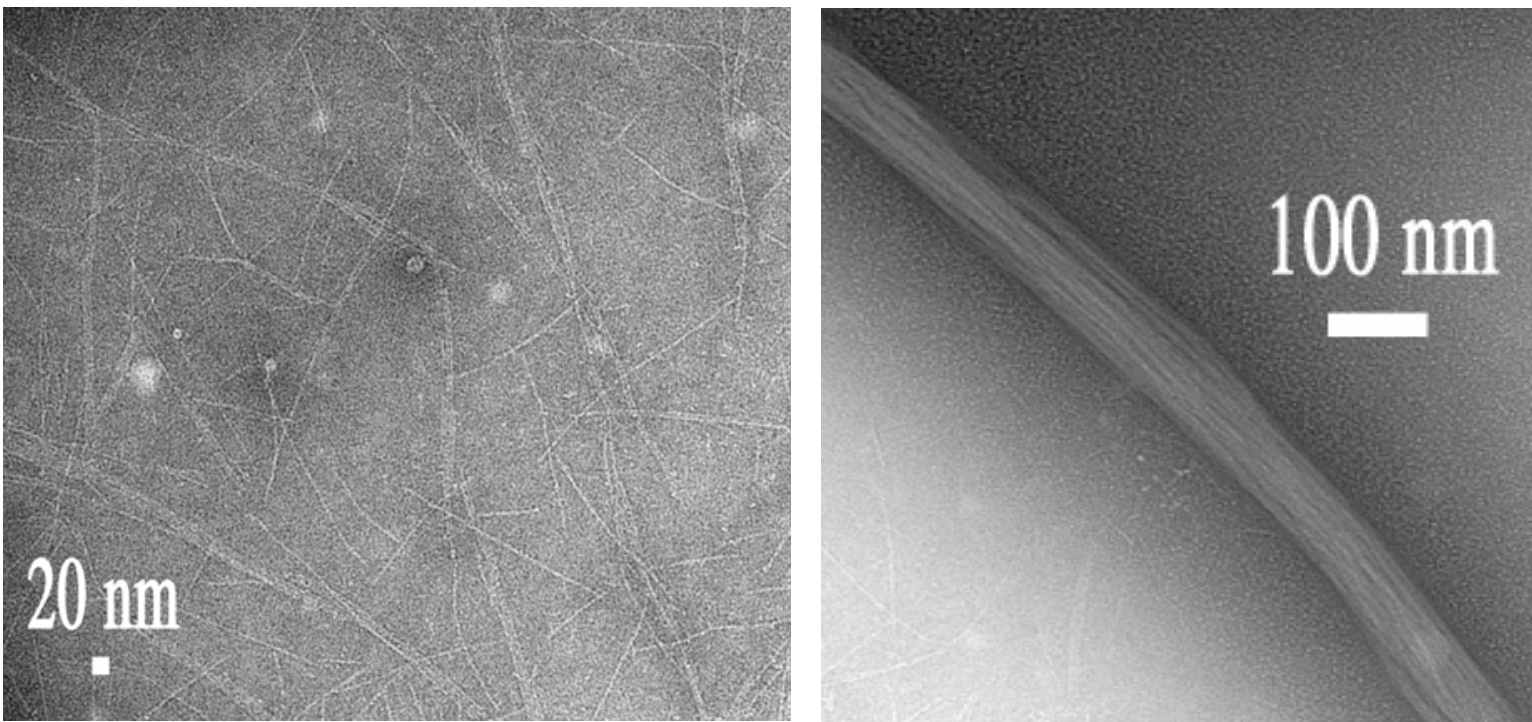

Fig. 2. Negative stain TEM showing $<4 \mathrm{~nm}$ coiled-coil filaments (left) and $>100 \mathrm{~nm}$ helical fibril ropes (right). 